\title{
Deviant trajectories of cortical maturation in 22q11.2 deletion syndrome (22q11DS): A cross-sectional and longitudinal study
}

\author{
Marie Schaer ${ }^{\mathrm{a}, \mathrm{b}, *}$, Martin Debbané ${ }^{\mathrm{a}}$, Meritxell Bach Cuadra ${ }^{\mathrm{b}}$, Marie-Christine Ottet ${ }^{\mathrm{a}}$, \\ Bronwyn Glaser ${ }^{\mathrm{a}}$, Jean-Philippe Thiran ${ }^{\mathrm{b}}$, Stephan Eliez ${ }^{\mathrm{a}}$ \\ a Service Médico-Pédagogique, Department of Psychiatry, Geneva Faculty of Medicine, 1 rue David Dufour, P.O. Box 50, CH-1211 Geneva 8, Switzerland \\ b Signal Processing Laboratory, Swiss Federal Institute of Technology, CH-1015 Lausanne, Switzerland
}

\section{A R T I C L E I N F O}

Article history:

Received 11 March 2009

Received in revised form 15 July 2009

Accepted 13 September 2009

\section{Keywords:}

Velo-cardio-facial

Schizophrenia

Freesurfer

Cortical thickness

COMT

\begin{abstract}
A B S T R A C T
22q11.2 deletion syndrome (22q11DS) is associated with an increased susceptibility to develop schizophrenia. Despite a large body of literature documenting abnormal brain structure in 22q11DS, cerebral changes associated with brain maturation in 22q11DS remained largely unexplored. To map cortical maturation from childhood to adulthood in 22q11.2 deletion syndrome, we used cerebral MRI from 59 patients with 22q11DS, aged 6 to 40, and 80 typically developing controls; three year follow-up assessments were also available for 32 patients and 31 matched controls. Cross-sectional cortical thickness trajectories during childhood and adolescence were approximated in age bins. Repeated-measures were also conducted with the longitudinal data. Within the group of patients with 22q11DS, exploratory measures of cortical thickness differences related to COMT polymorphism, IQ and schizophrenia were also conducted. We observed deviant trajectories of cortical thickness changes with age in patients with 22q11DS. In affected preadolescents, larger prefrontal thickness was observed compared to age-matched controls. Afterward, we observed greater cortical loss in 22q11DS with a convergence of cortical thickness values by the end of adolescence. No compelling evidence for an effect of COMT polymorphism on cortical maturation was observed. Within 22q11DS, significant differences in cortical thickness were related to cognitive level in children and adolescents, and to schizophrenia in adults. Deviant trajectories of cortical thickness from childhood to adulthood provide strong in vivo cues for a defect in the programmed synaptic elimination, which in turn may explain the susceptibility of patients with 22q11DS to develop psychosis.
\end{abstract}

(c) 2009 Elsevier B.V. All rights reserved.

\section{Introduction}

22q11.2 deletion syndrome (22q11DS) is a neurogenetic condition which draws particular interest as a model to

\footnotetext{
* Corresponding author. Service Médico-Pédagogique, 1 rue David Dufour, P.O. Box 50, CH-1211 Geneva 8, Switzerland. Tel.: +41 2238867 31; fax: +41 22388 6769.

E-mail addresses: marie.schaer@unige.ch (M. Schaer), martin.debbane@unige.ch (M. Debbané), meritxell.bach@epfl.ch (M. Bach Cuadra), Marie-Christine.Ottet@unige.ch (M.-C. Ottet), bronwyn.glaser@unige.ch (B. Glaser),jp.thiran@epfl.ch (J.-P. Thiran), stephan.eliez@unige.ch (S. Eliez).
}

understand the pathogenesis of schizophrenia (Murphy and Owen, 2001). A variety of genes have been identified in the $3 \mathrm{Mb}$ deletion associated with 22q11DS, among which haploinsufficiency of the catechol-O-methyltransferase (COMT) gene has received a large emphasis (Gothelf et al., 2008). From the point of view of structural neuroimaging, a large body of literature is aimed at delineating the cerebral phenotype in these individuals at risk for developing schizophrenia. Numerous studies have reported specific patterns of volumetric alterations in children and adults with the syndrome (Schaer and Eliez, 2007; Gothelf et al., 2008). However, between-group comparisons in sample with restrained age ranges only provide snapshots over the dynamic unfolding of structural brain changes with age. As a 
result, the question as to how the brain matures throughout life in individuals affected by 22q11DS remains scarcely documented. Providing a comprehensive picture of the dynamic of brain maturation from childhood to adulthood in the syndrome is all the more important, if theories argue that schizophrenia involves a disruption in the cortical maturational processes during adolescence (Hoffman and Dobscha, 1989; Keshavan et al., 1994).

To study structural brain maturation in vivo using MRI, cortical thickness emerges as the ideal technique among other methods. The typical curvilinear trajectories of thickness changes with age share strikingly similar temporal and regional similarities with the progression of synaptic pruning as observed postmortem, confirming the potential of cortical thickness to reliably identify the brains critical development periods (Sowell et al., 2003; Gogtay et al., 2004; Shaw et al., 2006a, 2008; Thompson et al., 2004). Moreover, cortical thickness studies have an exquisite resolution, allowing for the identification of local alterations with high precision. Consequently, a growing amount of studies apply thickness measurement as an index of development in patients with various conditions, such as attention deficit/hyperactivity disorder (ADHD) (Shaw et al., 2007a, 2006b), autism (Chung et al., 2005; Hadjikhani et al., 2007; Hardan et al., 2006), or schizophrenia (Thompson et al., 2004; Greenstein et al., 2006; Kuperberg et al., 2003). In 22q11DS, previous studies by Bearden and colleagues in 21 affected children and adolescents compared to 13 controls reported thinner superior parietal, right parieto-occipital and ventro-medial occipito-temporal cortices (Bearden et al., 2007; Bearden et al., 2009). However, these studies did not account for cortical thickness changes with age, warranting further exploration of the dynamic of cortical thickness over a more encompassing age range.

In the present study, we exploited neuroimaging data from patients with 22q11DS aged 6 to 40 who have been participating in our research project since 2001 (Debbané et al., 2006; Glaser et al., 2006; Schaer et al., 2006). As supported by longitudinal volumetric observations (Gothelf et al., 2005), we expected that patients will show a more intense thinning in the prefrontal regions during adolescence than controls.

In subsequent analyses, we also explored the effect of specific parameters on cortical thickness within the syndrome. First, we explored the possibility that patients with 22q11DS will show different cortical thickness depending of their genetic polymorphism on the $\mathrm{COMT}^{158}$ (Val vs. Met) allele. Patients carrying the low-activity Met allele may be exposed to unusually high levels of prefrontal dopamine that impair their cognitive functioning, a hypothesis which is supported by previous studies identifying the Met allele as a risk factor for poorer outcome in 22q11DS (Gothelf et al., 2005; Zinkstok et al., 2008; van Amelsvoort et al., 2008). Second, we subdivided our sample of patients with 22q11DS into children and adolescents (below 18 years old), and adults. In the younger subgroup, we investigated cortical thickness differences related to IQ. In accordance with previous observations of increased gray matter density or thicker cortexin high-functioning individuals compared to those with lower IQ (Shaw et al., 2006a; Wilke et al., 2003; Frangou et al., 2004; Narr et al., 2007; Fjell et al., 2006), we expected that patients with poorer performances will show thinner cortices than those with higher IQ scores. Finally, in the adult subsample, we searched for cortical thickness differences related to schizophrenia. As observed in non-syndromic adults with schizophrenia (Greenstein et al., 2006; Kuperberg et al., 2003), we expected that a thinner cortex in the frontal and temporal regions will characterize patients with schizophrenia compared to those without.

\section{Methods}

\subsection{Participants}

Patients with 22q11DS were recruited through announcement in regional parents associations. Fifty-nine patients with confirmed 22q11.2 deletion were included in the study (35F/ $24 \mathrm{M}$ ), with an average age of $15.9 \pm 8.9$ (see Table 1 ). Using the Wechsler Full Scale IQ the group of patients had an average IQ of $69.0 \pm 12.0$. Hemizygosity for either the $\mathrm{COMT}^{158}$ Met or the $\mathrm{COMT}^{158}$ Val allele was determined by polymerase chain reaction with the restriction enzyme NlaIII (Lachman et al., 1996). At the time of evaluation, 7 patients received antipsychotic treatment (age range: 19.6-36.6), 3 received methylphenidate (age range: 6.4-12.6) and one received antiepileptic treatment ( 11.5 years old).

Eighty healthy participants were recruited through a newsletter distributed at public schools in Geneva and in the community $(44 \mathrm{~F} / 36 \mathrm{M})$. Subjects with a history of past or present neurological or psychiatric disorders were excluded. The control group had a mean age of $15.9 \pm 8.4$ and an average IQ of 111.712.8. Written informed consent was received from participants and their parents (for subjects younger than 18 years old), under protocols approved by the Institutional Review Board of Geneva University School of Medicine.

Thirty-two patients who were younger than 18 years old at the first evaluation participated at follow-up examination (interval: 3.10 .3 years). The longitudinal subgroup of patients $(19 \mathrm{~F} / 13 \mathrm{M})$ had a mean age of $11.4 \pm 3.5$ at time 1 (T1) and $14.5 \pm$ 3.6 at time 2 (T2). The average IQ for this subgroup did not differ at T1 $(70.7 \pm 10.9)$ and T2 (71.6 \pm 12.8$)$. Thirty-one healthy participants $(20 \mathrm{~F} / 11 \mathrm{M})$ matched for age also participated in the follow-up examination (interval: $3.0 \pm 0.3$ years). The longitudinal subgroup of controls had a mean age of $11.1 \pm 3.6$ at T1 and $14.2 \pm 3.6$ at T2, with an average IQ of $112.7 \pm 12.4$ at T1 and $108.4 \pm 12.3$ at $\mathrm{T} 2$.

Table 1

Repartition of the participants in the different age bins.

\begin{tabular}{|c|c|c|c|c|}
\hline & \multicolumn{2}{|c|}{ Cross-sectional } & \multicolumn{2}{|c|}{ Longitudinal } \\
\hline & Control & $22 q 11 D S^{a}$ & Control & $22 q 11 D S^{a}$ \\
\hline 6 to 9 & 16 & $15(0 / 0)$ & 11 & $9(0 / 0 ; 0 / 3)$ \\
\hline 9 to 12 & 22 & $11(0 / 4)$ & 9 & $11(0 / 4 ; 0 / 6)$ \\
\hline 12 to 15 & 8 & $10(0 / 2)$ & 5 & $8(0 / 1 ; 0 / 1)$ \\
\hline 15 to 18 & 7 & $4(0 / 3)$ & 6 & $4(0 / 3 ; 2 / 3)$ \\
\hline $18+$ & 27 & $19(6 / 15)$ & & \\
\hline Total & 80 & $59(6 / 24)$ & 31 & $32(0 / 8 ; 2 / 13)$ \\
\hline
\end{tabular}

The right part of the table details the subsample who participated in the follow-up examination.

a In the patients' columns, psychiatric statuses are described in brackets as follows: "(number of schizophrenic patients/number of patients presenting either hallucinations or delusions or both)". For the longitudinal subgroups, psychiatric characteristics are presented similarly, but with "(status at Time 1; status at Time 2)". 


\subsection{Imaging}

Coronal cerebral MRI was acquired using a Philips 1.5 T Intera scanner with a 3D volumetric pulse sequence using the following scan parameters: $\mathrm{TR}=35 \mathrm{~ms}, \mathrm{TE}=6 \mathrm{~ms}$, flip angle $=45^{\circ}$, $\mathrm{NEX}=1$, matrix size $=256 \times 192$, field of view $=24 \mathrm{~cm}^{2}$, slice thickness $=1.5 \mathrm{~mm}, 124$ slices.

Images were imported into the software FreeSurfer (http:// surfer.nmr.mgh.harvard.edu) to reconstruct accurate cortical surface models. The algorithms used for cortical thickness estimation were previously validated against manual delineation on MRI sections (Kuperberg et al., 2003) and postmortem brains (Rosas et al., 2002). Further, cortical thickness measurements have been proven reliable, independently of scanner manufacturer or field strength (Han et al., 2006).

Fully automated image processing included resampling into cubic voxels, intensity normalization and skull stripping. Subsequent surface reconstructions used deformation algorithms based on the local intensity value, geometrical and topological constraints (Dale et al., 1999; Fischl et al., 2001). The final threedimensional cortical surfaces include the white (gray-white boundary) and the pial (gray-CSF interface) surfaces. Cortical thickness was measured in the native space of the images, as the shortest distance between the white and pial surfaces (Fischl and Dale, 2000). As a result, cortical thickness values with a submillimeter accuracy were available at more than 150,000 points over each hemisphere.

\subsection{Statistical analyses}

\subsubsection{Cross-sectional analyses}

Statistical analyses employed a general linear model (GLM) to estimate the effect of diagnosis, age and diagnosis by age at each cortical point. Cortical thickness changes with age were fitted using a linear model. A false discovery rate at FDR $<0.05$ was employed to correct for Type 2 errors related to multiple comparisons. Interactions for gender were not included in the final model because they did not contribute significantly to the explanatory power of the model across the cortical surface (after employing a FDR correction).

To refine the trajectories of cortical changes with age, we further subdivided the sample of subjects into 5 age bins, as detailed in Table 1 . The 3 year interval was chosen in order to keep a sufficient number of subjects within each age bin, while keeping an appropriate temporal resolution in the period where the most cortical changes are known to occur. Moreover, 3 years corresponded exactly to the interval between the two timepoints for longitudinal analyses. Within each age bin, a GLM was employed to assess the effect of diagnosis at each vertex.

\subsubsection{Longitudinal analyses}

Mixed effect models, which would have been the gold standard technique to combine cross-sectional and longitudinal data, commonly encompass more than 500 scans (Shaw et al., 2006a, 2006b, 2007a, 2007b, 2008) or 3-4 time-points (Gogtay et al., 2004; Greenstein et al., 2006). Given the limited size of our sample, we preferred the use of repeated-measures at each cortical vertex, to identify significant differences in thickness changes over the 3 year period. The repeated-measures were separately conducted on the subgroups that were below 9 of age at Time 1 , and those that were between 9 and 18 at Time 1 . This subdivision was based on a priori cross-sectional analyses showing distinct thickness changes in age bins below and above 9 years old (see Fig. 2a).

\subsubsection{COMT polymorphism}

Of the 59 patients, information about the COMT ${ }^{158} \mathrm{Val} / \mathrm{Met}$ status was not available for 2 of them. The remaining 57 showed the following COMT polymorphism: 29 patients had the Met allele (18F/11M; mean age: $16.8 \pm 8.8$; average IQ: $68.7 \pm 10.7)$ and 28 had the Val allele $(16 \mathrm{~F} / 12 \mathrm{M}$; mean age: $15.4 \pm 9.3$; average IQ: $68.3 \pm 13.1)$. The Val and Met subgroups did not differ neither in age distribution $(p=0.541)$, nor in IQ $(p=0.907)$. To determine the effect of COMT polymorphism on cortical thickness, a GLM was applied at each vertex, modeling the effect of COMT and age. Furthermore, repeatedmeasures were also conducted on the longitudinal subsample of 32 patients ( $15 \mathrm{Met} / 17 \mathrm{Val}$ ) to evidence differences in cortical maturation associated with COMT polymorphism. Given previous evidence for an interaction of COMT by gender during brain development in 22q11DS (Kates et al., 2006), gender was introduced as an independent variable in the longitudinal analysis.

\subsubsection{Within group factors: IQ and schizophrenia}

In patients below 18 years old, we defined a low performing subgroup with all children and adolescents who had an established mental delay, as defined by an IQ lower or equal to 65 ( $n=13,7 \mathrm{~F} / 6 \mathrm{M}$, FSIQ: $58.9 \pm 5.5$, range: $44-64)$. Twentyone children and adolescents scored above 75 (13F/8M, FSIQ: $81.1 \pm 4.6$, range: $75-91)$ and constituted the high performing subgroup. Both groups had similar average age (low: $11.2 \pm 4.5$; high: $10.2 \pm 4.0, p=0.371)$. A GLM was applied to assess the effect of group, age and age by group interaction on cortical thickness at each vertex.

Then, cortical thickness changes associated with schizophrenia were examined in the 46 individuals who were older than 18 years old, namely 27 controls $(14 \mathrm{~F} / 13 \mathrm{M}$, mean age: $26.0 \pm$ 5.9), 6 schizophrenic patients (3F/3M, mean age: $31.6 \pm 4.1)$ and 13 non-schizophrenic adults with 22q11DS (8F/5M, mean age: $24.8 \pm 6.2$ ). There was a trend for a significant difference in age distribution between the 3 subgroups $(F=2.944, p=0.063)$. Post-hoc analyses demonstrated no age difference between the control and non-schizophrenic groups $(p=0.843)$ or between the control and schizophrenic groups $(p=0.113)$. However, a trend for a significant difference between the non-schizophrenic and schizophrenic groups $(p=0.073)$. Because of this age difference, no age by group interaction could be assessed; rather a GLM assessed the effect of group and age on cortical thickness at each vertex.

\section{Results}

\subsection{Cross-sectional analyses}

ANOVA comparing mean thickness values over the whole sample revealed significantly higher thickness in patients $(2.67 \pm$ $0.20 \mathrm{~mm}$ ) than controls $(2.60 \pm 0.16 \mathrm{~mm} ; F=5.934, p=0.016)$. The effect of diagnosis on mean thickness became even more significant when covarying for age $\left(F_{2,136}=17.785, p<0.001\right)$, or age and gender $\left(F_{3,135}=17.701, p<0.001\right)$. Statistical cortical maps revealed that regions of thicker cortex were relatively 
widespread, touching extensively the frontal regions (Fig. 1a). At the same time than increased thickness, a significant age by diagnosis interaction was observed, with a steeper slope of cortical thickness reduction with age in patients than controls (Fig. 1b).

Fig. 2a illustrates the profile of mean cortical thickness over the different age bins, while cortical maps depict the detailed distribution of significant thickness differences between groups. In the youngest bin, patients show significantly larger thickness values than controls in the left hemisphere $(F=6.541, p=0.016)$ and a trend for a thicker cortex in the right hemisphere $(F=3.3387, p=0.076)$. At this age, widely distributed small clusters of thicker cortex are observed in patients. In the 9 to 12 age bin, significantly greater thickness is observed in both hemispheres (right: $F=15.490, p<0.001$; left: $F=12.076$, $p=0.002$ ), mostly driven by thicker frontal cortex in patients than controls. In the 12 to 15 age bin, higher average thickness values are still observed in both hemispheres (right: $F=13.496$, $p=0.002$; left: $F=9.616, p=0.007$ ), that appear to be mostly driven by thicker cortex in the dorsal frontal regions. In the 15 to 18 bin, we do not observe any differences in average thickness (right: $F=1.186, p=0.302$; left: $F=1.618, p=0.232$ ). Although the absence of significant difference may be driven by the smallest sample size, the absence of significant average thickness difference in the subsequent adult bin (right: $F=0.050, p=0.825$; left: $F=0.326, p=0.571$ ) further confirms the disappearance of the thicker cortex observed in younger patients with 22q11DS compared to controls.

\subsection{Longitudinal analyses}

As depicted in Fig. 2b, we were able to confirm the trajectories of cortical thickness changes identified with cross-sectional design using longitudinal repeated-measures. In the younger subgroup, we observed clusters where cortical thickness loss over the 3 year period was significantly larger in controls than in patients. For instance in several clusters of the frontal lobe, cortical thinning was shown in control participants whereas patients showed almost no thickness changes or even slight cortical thickening (see plots A to D on Fig. 2b). Contrarily, in the older subgroups, we identified widespread clusters of significantly greater cortical loss in patients compared to controls.

\subsection{COMT polymorphism}

No significant effect on COMT polymorphism was observed on mean cortical thickness values (Met: $2.630 .21 \mathrm{~mm}$, Val: $2.700 .20 \mathrm{~mm} ; F=1.732, p=0.194)$. As shown in Fig. 3, the statistical maps also did not reveal a compelling intensity and direction of changes associated with COMT polymorphism, neither using the cross-sectional nor the longitudinal analyses. Indeed, cortical thickness differences between subgroups were observed in both directions and were only of modest significance.

\subsection{Within group: IQ and schizophrenia}

In the patients with 22q11DS below 18 years old, the cortical maps demonstrated numerous clusters of significantly thinner cortex in low performing compared to high performing subgroup (Fig. 4). Namely, thinner cortex in the medial frontal gyri bilaterally, the superior and inferior right frontal gyri, and the left superior temporal sulcus were seen in the low performing subgroup. No group by age interaction was observed.

In adult participants (Fig. 5), statistical maps of cortical thickness difference demonstrate that (1) patients without schizophrenia show a combination of increased and decreased thickness compared to healthy participants; (2) schizophrenic patients show extended regions of cortical thinning, as compared to healthy controls and to non-schizophrenic patients with 22q11DS. Among the region in which thinnest cortex was associated with schizophrenia, arrow heads on Fig. 5 at the right fusiform/lingual region and at the left superior frontal gyrus depict reduction in schizophrenic patients compared to both the controls and the non-schizophrenic patients. Thin arrows at the right middle frontal gyrus and the left superior temporal sulcus illustrate an intriguing pattern of thickness change, with normal thickness in patients with schizophrenia, whereas aberrantly high thickness in these regions was observed in patients without schizophrenia.

\section{Discussion}

The work presented here uses cortical thickness to quantify the course of cerebral maturation over a wide age range in 22q11.2 deletion syndrome. When comparing the entire

\section{b Age by diagnosis interaction}

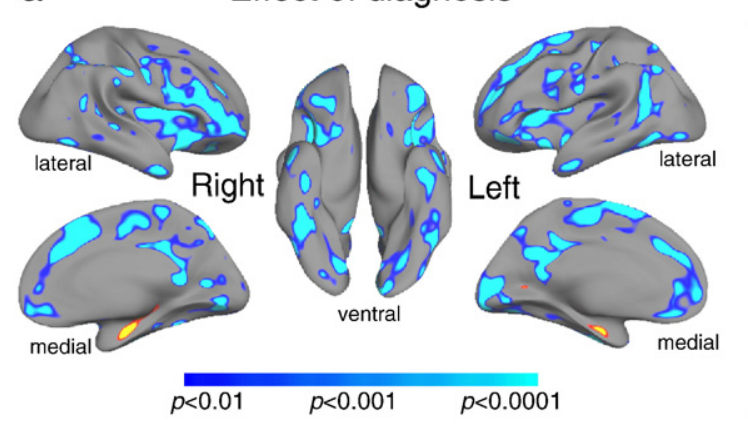

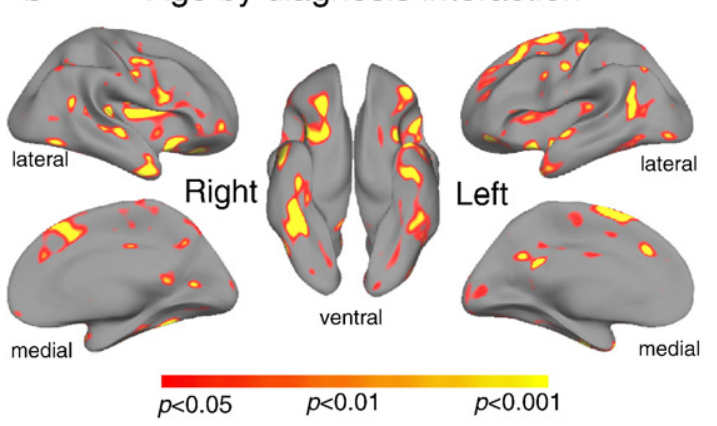

Fig. 1. Vertex-wise comparisons of cortical thickness between groups (cross-sectional). (a) When comparing the entire sample, we observe extensive areas of thicker cortex in patients with 22q11DS compared to controls (in blue). Small regions of thinner cortex in 22q11DS are shown around the bilateral entorhinal and parahippocampal regions. (b) Clusters in red/yellow show regions of significant age by diagnosis interaction, meaning that the regression line of cortical thickness changes with age shows a significantly steeper slope in patients with 22q11DS compared to controls. 


\section{Right Hemisphere}

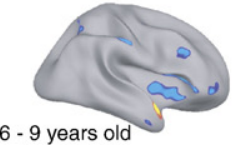

$9-12$ years old

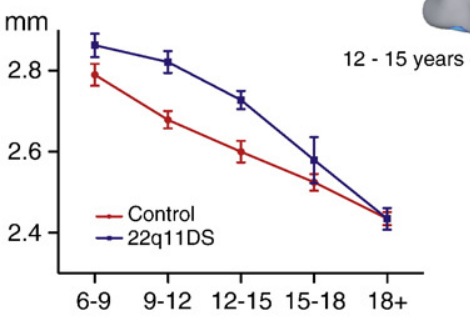

$\begin{array}{lllll}6-9 & 9-12 & 12-15 & 15-18 & 18+\end{array}$

\section{Left Hemisphere}

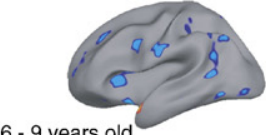

$6-9$ years old

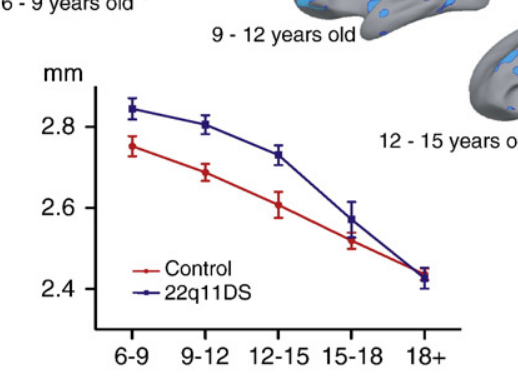

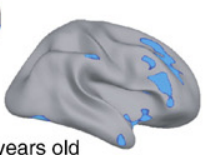

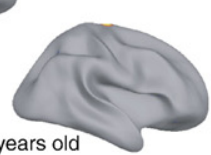

$p<0.0001$

more than 18

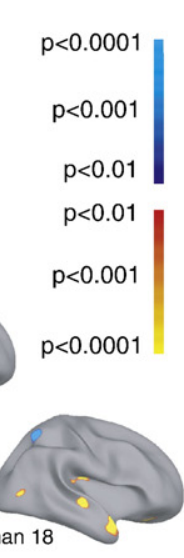

$15-18$ years old
(Longitudinal)

1) Reduced thinning in preadolescents
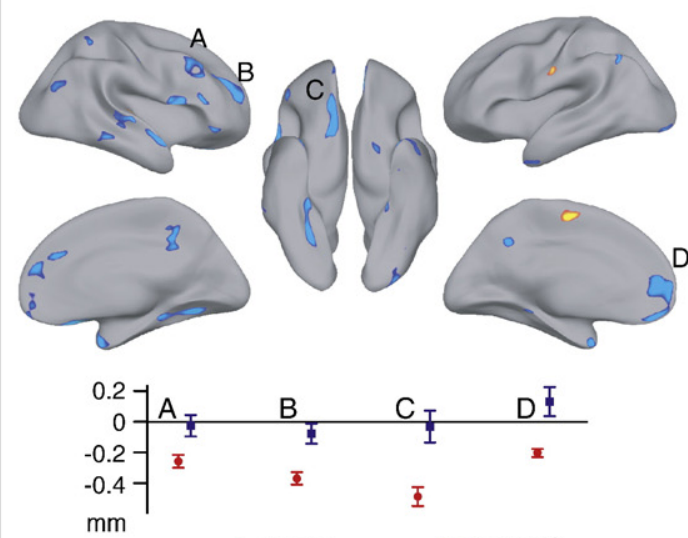

$\rightarrow$ Control $\rightarrow$ 22q11DS

2) Increased thinning during adolescence

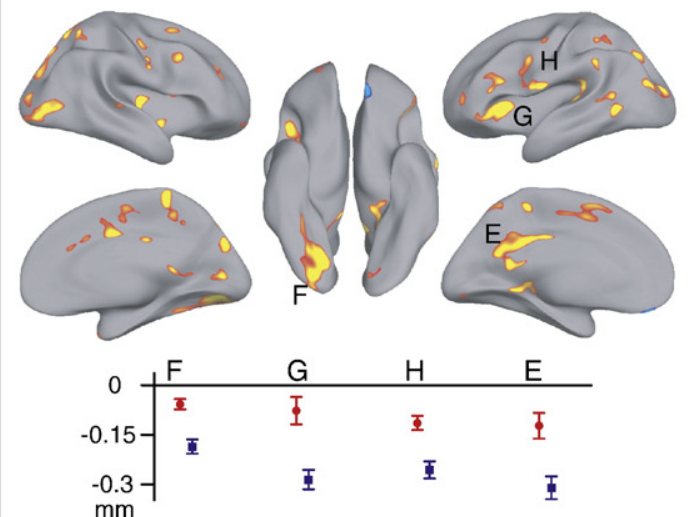

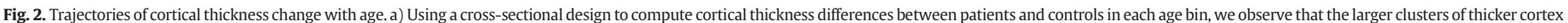

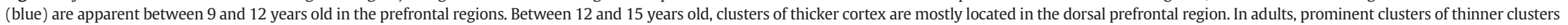

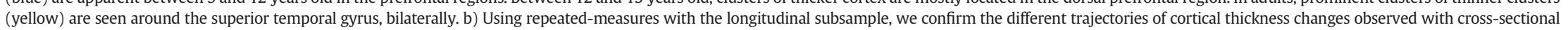

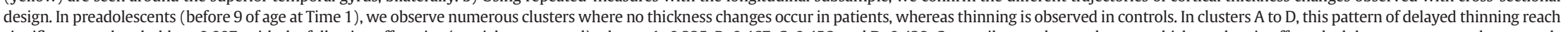

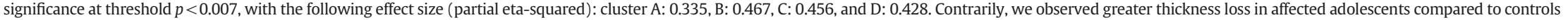
(older than 9 at Time 1 ). This larger thinning with age in patients compared to controls is significant at $p<0.002$ and with the following partial eta-squared: cluster F: 0.342 , G: 0.215 , H: 0.285 , and E: 0.239 . 


\section{Effect of COMT polymorphism}

a On cortical thickness (cross-sectional)

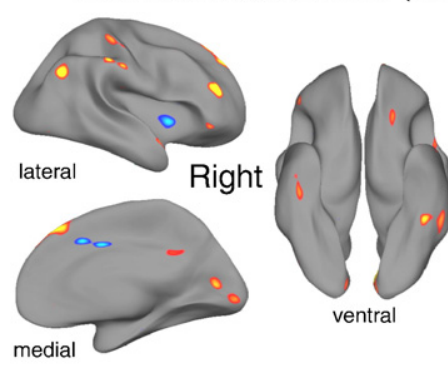

b On cortical maturation (longitudinal)

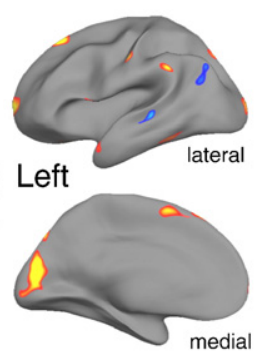

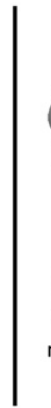

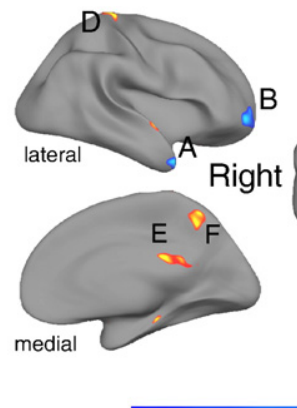

$p<0.05$
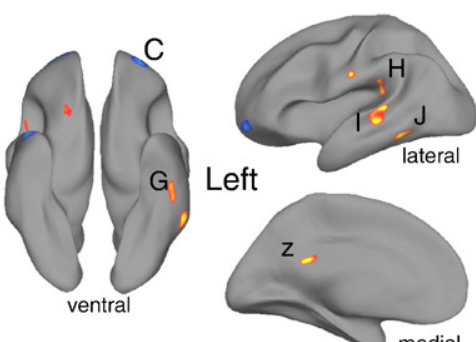

Left

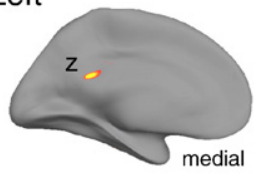

$p<0.01 \quad p<0.001$

Fig. 3. Effect of COMT polymorphism on cortical thickness within 22q11DS. a) Apart from a cluster of thinner cortex at the left cuneus in patients with Met compared to Val allele (in yellow), only modest clusters of significant differences associated with COMT polymorphism are observed. b) Using repeated-measures in the longitudinal subsample, no compelling evidence for an unequivocal age by COMT polymorphism interaction is shown. Namely, clusters in blue illustrate larger thinning in Val compared to Met reaching the significance of $p<0.023$ (partial eta-squared: A: 0.196, B: 0.225, C: 0.161). Clusters in yellow show greater cortical loss in Met compared to Val with significance of $p<0.022$ (partial eta-squared: D: 0.205, E: 0.279, F: 0.256, G: 0.234, H: 0.164, I: 0.297, J: 0.244, K: 0.181).

sample of patients to age-matched controls, we observe several clusters of thicker cortex (Fig. 1a). The observation of thicker cortex partially modifies the classical view that patients with 22q11DS consistently show differences in the reduced direction. Indeed, volumetric studies have always reported reduced cortical volume in the syndrome (Eliez et al., 2000; Kates et al., 2004, 2001; Simon et al., 2005; van Amelsvoort et al., 2001). If cortical volume is undeniably the product of its surface and thickness, surface changes are more susceptible to result in volumetric changes than thickness alterations (Im et al., 2008). Thus, the drastic cortical surface reduction that we observed in previous studies (Schaer et al., 2006, 2008) may better predict the decreased volume than subtle thickness changes associated with maturation. The present observation of thicker cortex in preadolescents with 22q11DS also contrasts with observations of thinner cortex in children aged from 8 to 17 years old (Bearden

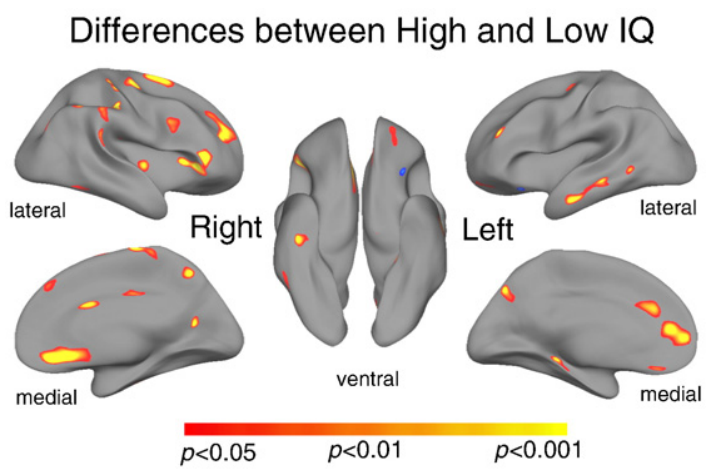

Fig. 4. Differences in cortical thickness related to cognitive abilities within 22q11DS. Clusters in red/yellow depict regions where children and adolescents with 22q11DS who score above 75 on the IQ tests show larger thickness values than those with IQ below 65. This association between thicker cortex and better performances seems to hold true at each age, as no significant clusters of group by age interaction were evidenced at $p<0.05$ (statistical maps not shown). et al., 2007, 2009). However, methodological differences between these studies and ours, such as scaling of the brain, divergences in cortical thickness measurements, and absence of correction for an effect of age in the statistical analyses, may account for a different direction of the results.

From childhood to adulthood, we observed distinct patterns of thickness alterations in 22q11DS, corroborating the hypothesis that the dynamic rather than the absolute value of cortical thickness is more relevant during brain development (Shaw et al., 2006a, 2007a). In the youngest patients, we observed only minimal thickness changes, followed by a significantly thicker cortex in preadolescents with 22q11DS, and by a subsequent disappearance of the changes by the end of adolescence. As illustrated in Fig. 2a, the transient thickening mostly affecting the frontal regions of affected patients supports a delay in the normal process of cortical thinning with age. Retarded onset of frontal cortical thinning was also recently observed in ADHD (Shaw et al., 2007a). But contrarily to their observation of parallel trajectories of cortical thickness change in ADHD and controls, the delay observed here in 22q11DS is associated with deviant trajectories of cortical changes. Indeed, the significant age by diagnosis interaction (Fig. 1b), the absence of significant thickening by the end of adolescence (Fig. 2a) and the longitudinal analyses (Fig. 2b), all provide compelling evidence for a greater thickness loss after the age of 9 in 22q11DS. An exaggerated rate of thinning in 22q11DS may reflect a disturbance in the maturational processes occurring during adolescence, namely a defect in the programmed synaptic elimination.

More than two decades ago, Feinberg proposed that a defect in pruning may be associated with schizophrenia (Feinberg, 1982), a theory which is corroborated by the observation of decreased neuropil on neuropathological examinations (Arnold, 1999). In neural networks models, hypoconnectivity or misconnectivity caused by excessive pruning has been suggested to decrease functional efficiency and to trigger abnormal perceptions comparable to hallucinations (Hoffman and McGlashan, 
a Controls vs non-schizophrenic patients
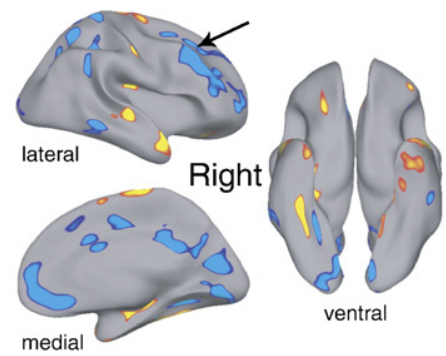

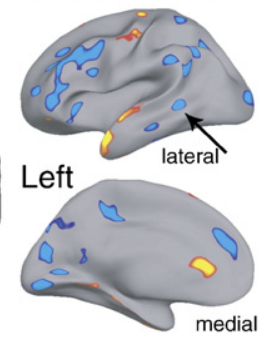

medial b Controls vs schizophrenic patients
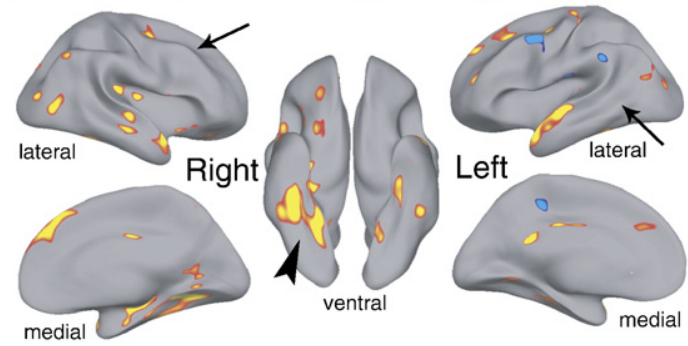

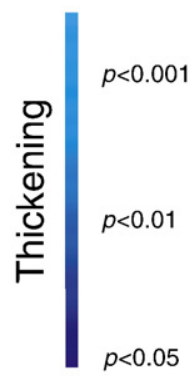

C Non-schizophrenic vs schizophrenic patients
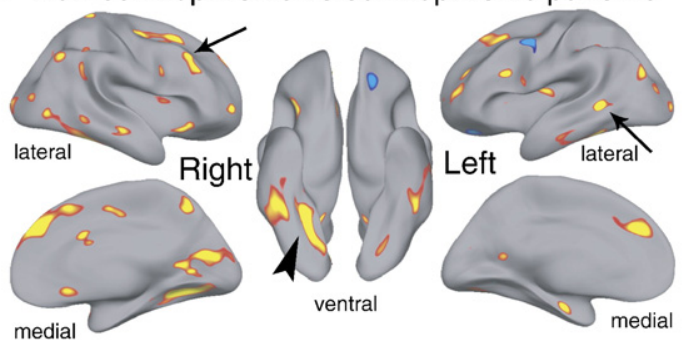

$p<0.001$

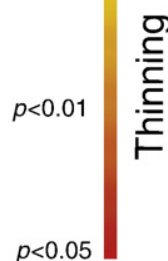

Fig. 5. Cortical thickness differences related to schizophrenia in adults. In the controls vs. non-schizophrenic patients, clusters in blue demonstrate thicker cortex in patients than controls, whereas red/yellow clusters show thinner cortex in patients compared to controls. When controls are compared to schizophrenic patients, only clusters of thinner cortex (red/yellow) are found in patients. Finally, the comparison within 22q11DS reveals mostly areas of cortical thinning (red/yellow) in schizophrenic compared to non-schizophrenic patients.

1997; Hoffman and McGlashan, 2006; McGlashan and Hoffman, 2000). However, the association between aberrant pruning and schizophrenic symptomatology remains difficult to prove in vivo, e.g. using EEG (Micheloyannis et al., 2006), fMRI (Liu et al., 2008), or DTI (Kubicki et al., 2007). In this study, we observed that adult patients with 22q11DS and without schizophrenia demonstrate preserved thickness as compared to age-matched controls. Contrarily, patients with schizophrenia show a thinner cortex as compared to controls and to patients without. Together, these observations provide a strong support that excessive cortical thinning during adolescence is selectively associated with the onset of schizophrenia. A distinct neuroanatomical phenotype in patients with and without schizophrenia is in agreement with previous cross-sectional volumetric studies in 22q11 deletion syndrome (van Amelsvoort et al., 2004). More recently, a longitudinal study documented that a more drastic the prefrontal reduction was associated with the emergence of psychotic symptoms (Gothelf et al., 2005). If future studies following the course of cortical changes during adolescence confirm that exaggerated pruning is specifically seen in individuals developing schizophrenic symptoms, the search for factors interacting with synaptic pruning may serve as a guide for future targeted preventions.

\section{Role of funding source}

This research and the clinical research structure were supported by Swiss National Research Funds (grants 3200-063135, 3232-063134, and PP00B102864 to S.E.) and by NARSAD Institute. Individual support to Dr Schaer was provided by a Swiss National Research Fund for carrier development (grant 323500-111165). The Center for Biomedical Imaging of the Geneva - Lausanne Universities and the EPFL, as well as the foundations Leenaards and Louis-Jeantet (www.cibm.ch), provided support for acquiring the cerebral MRI images. The sponsors of the study had no role in the study design, collection, analysis, interpretation of the data, writing of the report, and in the decision to submit the paper for publication.

\section{Contributors}

M. Schaer, M. Debbane, B. Glaser and S. Eliez designed the study; B. Glaser, M. Debbane and S. Eliez acquired the data, including magnetic resonance imaging and clinical assessments of the study participants; M. Schaer and M.-C. Ottet analyzed the data; M. Schaer and B. Glaser undertook the statistical analysis; M. Bach Cuadra and J.-P. Thiran supervised the technical aspects of the data analysis and the statistical procedures; M. Schaer and M. Debbane wrote the first draft of the manuscript; all authors contributed to the interpretation of the results and the writing of the manuscript. All authors have approved the final manuscript.

\section{Conflict of interest}

All authors report no competing interests.

\section{Acknowledgments}

The authors would like to thank all the families who participated in this research project since 2001. Further thanks go to François Lazeyras and Frank Henry for their help in scanning the subjects.

\section{References}

Arnold, S.E., 1999. Neurodevelopmental abnormalities in schizophrenia: insights from neuropathology. Dev. Psychopathol. 11 (3), 439-456.

Bearden, C.E., van Erp, T.G., Dutton, R.A., Tran, H., Zimmermann, L., Sun, D., Geaga, J.A., Simon, T.J., Glahn, D.C., Cannon, T.D., Emanuel, B.S., Toga, A.W., Thompson, P.M., 2007. Mapping cortical thickness in children with 22q11.2 deletions. Cereb. Cortex 17 (8), 1889-1898.

Bearden, C.E., van Erp, T.G., Dutton, R.A., Lee, A.D., Simon, T.J., Cannon, T.D., Emanuel, B.S., McDonald-McGinn, D., Zackai, E.H., Thompson, P.M., 2009. Alterations in midline cortical thickness and gyrification patterns mapped in children with 22q11.2 deletions. Cereb. Cortex 19 (1), 115-126.

Chung, M.K., Robbins, S.M., Dalton, K.M., Davidson, R.J., Alexander, A.L., Evans, A.C., 2005. Cortical thickness analysis in autism with heat kernel smoothing. Neuroimage 25 (4), 1256-1265.

Dale, A.M., Fischl, B., Sereno, M.I., 1999. Cortical surface-based analysis. I. Segmentation and surface reconstruction. Neuroimage 9 (2), 179-194.

Debbané, M., Glaser, B., David, M.K., Feinstein, C., Eliez, S., 2006. Psychotic symptoms in children and adolescents with 22q11.2 deletion syndrome: neuropsychological and behavioral implications. Schizophr. Res. 84 (2-3), 187-193. 
Eliez, S., Schmitt, J.E., White, C.D., Reiss, A.L., 2000. Children and adolescents with velocardiofacial syndrome: a volumetric MRI study. Am. J. Psychiatry 157 (3), 409-415.

Feinberg, I., 1982. Schizophrenia: caused by a fault in programmed synaptic elimination during adolescence? J Psychiatr Res 17 (4), 319-334.

Fischl, B., Dale, A.M., 2000. Measuring the thickness of the human cerebral cortex from magnetic resonance images. Proc. Natl. Acad. Sci. U. S. A. 97 (20), 11050-11055.

Fischl, B., Liu, A., Dale, A.M., 2001. Automated manifold surgery: constructing geometrically accurate and topologically correct models of the human cerebral cortex. IEEE Trans Med Imaging 20 (1), 70-80.

Fjell, A.M., Walhovd, K.B., Reinvang, I., Lundervold, A., Salat, D., Quinn, B.T., Fischl, B., Dale, A.M., 2006. Selective increase of cortical thickness in high-performing elderly-structural indices of optimal cognitive aging. Neuroimage 29 (3), 984-994.

Frangou, S., Chitins, X., Williams, S.C., 2004. Mapping IQ and gray matter density in healthy young people. Neuroimage 23 (3), 800-805.

Glaser, B., Debbane, M., Hinard, C., Morris, M.A., Dahoun, S.P., Antonarakis, S.E., Eliez, S., 2006. No evidence for an effect of comt val158met genotype on executive function in patients with $22 \mathrm{q} 11$ deletion syndrome. Am. J. Psychiatry 163 (3), 537-539.

Gogtay, N., Giedd, J.N., Lusk, L., Hayashi, K.M., Greenstein, D., Vaituzis, A.C., Nugent, T.F., Herman, D.H., Clasen, L.S., Toga, A.W., Rapoport, J.L., Thompson, P.M., 2004. Dynamic mapping of human cortical development during childhood through early adulthood. Proc. Natl. Acad. Sci. U. S. A. 101 (21), 8174-8179.

Gothelf, D., Eliez, S., Thompson, T., Hinard, C., Penniman, L., Feinstein, C., Kwon, H., Jin, S., Jo, B., Antonarakis, S.E., Morris, M.A., Reiss, A.L., 2005. Comt genotype predicts longitudinal cognitive decline and psychosis in 22q11.2 deletion syndrome. Nat. Neurosci. 8 (11), 1500-1502.

Gothelf, D., Schaer, M., Eliez, S., 2008. Genes, brain development and psychiatric phenotypes in velo-cardio-facial syndrome. Dev. Disabil. Res. Rev. 14 (1), 59-68.

Greenstein, D., Lerch, J., Shaw, P., Clasen, L., Giedd, J., Gochman, P., Rapoport, J., Gogtay, N., 2006. Childhood onset schizophrenia: cortical brain abnormalities as young adults. J. Child. Psychol. Psychiatry 47 (10), 1003-1012.

Hadjikhani, N., Joseph, R.M., Snyder, J., Tager-Flusberg, H., 2007. Abnormal activation of the social brain during face perception in autism. Hum. Brain Mapp. 28 (5), 441-449.

Han, X., Jovicich, J., Salat, D., van der Kouwe, A., Quinn, B., Czanner, S., Busa, E., Pacheco, J., Albert, M., Killiany, R., Maguire, P., Rosas, D., Makris, N., Dale, A., Dickerson, B., Fischl, B., 2006. Reliability of MRI-derived measurements of human cerebral cortical thickness: the effects of field strength, scanner upgrade and manufacturer. Neuroimage 32 (1), 180-194.

Hardan, A.Y., Muddasani, S., Vemulapalli, M., Keshavan, M.S., Minshew, N.J., 2006. An mri study of increased cortical thickness in autism. Am. J. Psychiatry 163 (7), 1290-1292.

Hoffman, R.E., Dobscha, S.K., 1989. Cortical pruning and the development of schizophrenia: a computer model. Schizophr. Bull. 15 (3), 477-490.

Hoffman, R.E., McGlashan, T.H., 1997. Synaptic elimination, neurodevelopment, and the mechanism of hallucinated "voices" in schizophrenia. Am. J. Psychiatry 154 (12), 1683-1689.

Hoffman, R.E., McGlashan, T.H., 2006. Using a speech perception neural network computer simulation to contrast neuroanatomic versus neuromodulatory models of auditory hallucinations. Pharmacopsychiatry 39 (Suppl 1), 54-64.

Im, K., Lee, J.M., Lyttelton, O., Kim, S.H., Evans, A.C., Kim, S.I., 2008. Brain size and cortical structure in the adult human brain. Cereb. Cortex 18 (9), 2181-2191.

Kates, W.R., Burnette, C.P., Jabs, E.W., Rutberg, J., Murphy, A.M., Grados, M., Geraghty, M., Kaufmann, W.E., Pearlson, G.D., 2001. Regional cortical white matter reductions in velocardiofacial syndrome: a volumetric MRI analysis. Biol. Psychiatry 49 (8), 677-684.

Kates, W.R., Burnette, C.P., Bessette, B.A., Folley, B.S., Strunge, L., Jabs, E.W., Pearlson, G.D., 2004. Frontal and caudate alterations in velocardiofacial syndrome (deletion at chromosome 22q11.2). J. Child Neurol. 19 (5), 337-342.

Kates, W.R., Antshel, K.M., Abdulsabur, N., Colgan, D., Funke, B., Fremont, W., Higgins, A.M., Kucherlapati, R., Shprintzen, R.J., Apr 2006. A gender-moderated effect of a functional comt polymorphism on prefrontal brain morphology and function in velo-cardio-facial syndrome (22q11.2 deletion syndrome). Am. J. Med. Genet. B Neuropsychiatr. Genet. 141B (3), 274-280.

Keshavan, M.S., Anderson, S., Pettegrew, J.W., 1994. Is schizophrenia due to excessive synaptic pruning in the prefrontal cortex? The Feinberg hypothesis revisited. J. Psychiatr. Res. 28 (3), 239-265.

Kubicki, M., McCarley, R., Westin, C.F., Park, H.J., Maier, S., Kikinis, R., Jolesz, F.A., Shenton, M.E., 2007. A review of diffusion tensor imaging studies in schizophrenia. J. Psychiatr. Res. 41 (1-2), 15-30.

Kuperberg, G.R., Broome, M.R., McGuire, P.K., David, A.S., Eddy, M., Ozawa, F., Goff, D., West, W.C., Williams, S.C., van der Kouwe, A.J., Salat, D.H., Dale, A.M., Fischl, B., 2003. Regionally localized thinning of the cerebral cortex in schizophrenia. Arch. Gen. Psychiatry 60 (9), 878-888.
Lachman, H.M., Morrow, B., Shprintzen, R., Veit, S., Parsia, S.S., Faedda, G., Goldberg, R., Kucherlapati, R., Papolos, D.F., 1996. Association of codon 108/ 158 catechol-o-methyltransferase gene polymorphism with the psychiatric manifestations of velo-cardio-facial syndrome. Am. J. Med. Genet. 67 (5), 468-472.

Liu, Y., Liang, M., Zhou, Y., He, Y., Hao, Y., Song, M., Yu, C., Liu, H., Liu, Z., Jiang, T. 2008. Disrupted small-world networks in schizophrenia. Brain 131 (Pt 4), 945-961.

McGlashan, T.H., Hoffman, R.E., 2000. Schizophrenia as a disorder of developmentally reduced synaptic connectivity. Arch. Gen. Psychiatry 57 (7), 637-648.

Micheloyannis, S., Pachou, E., Stam, C.J., Breakspear, M., Bitsios, P., Vourkas, M., Erimaki, S., Zervakis, M., 2006. Small-world networks and disturbed functional connectivity in schizophrenia. Schizophr. Res. 87 (1-3), 60-66.

Murphy, K.C., Owen, M.J., 2001. Velo-cardio-facial syndrome: a model for understanding the genetics and pathogenesis of schizophrenia. Br. J. Psychiatry 179, 397-402.

Narr, K.L., Woods, R.P., Thompson, P.M., Szeszko, P., Robinson, D., Dimtcheva, T., Gurbani, M., Toga, A.W., Bilder, R.M., 2007. Relationships between IQ and regional cortical gray matter thickness in healthy adults. Cereb. Cortex 17 (9), 2163-2171.

Rosas, H.D., Liu, A.K., Hersch, S., Glessner, M., Ferrante, R.J., Salat, D.H., van der Kouwe, A., Jenkins, B.G., Dale, A.M., Fischl, B., 2002. Regional and progressive thinning of the cortical ribbon in huntington's disease. Neurology 58 (5), 695-701.

Schaer, M., Eliez, S., 2007. From genes to brain: understanding brain development in neurogenetic disorders using neuroimaging techniques. Child Adolesc. Psychiatr. Clin. N. Am. 16 (3), 557-579.

Schaer, M., Schmitt, J.E., Glaser, B., Lazeyras, F., Delavelle, J., Eliez, S., 2006. Abnormal patterns of cortical gyrification in velo-cardio-facial syndrome (deletion 22q11.2): an MRI study. Psychiatry Res. 146 (1), 1-11.

Schaer, M., Cuadra, M.B., Tamarit, L., Lazeyras, F., Eliez, S., Thiran, J.P., 2008. A surface-based approach to quantify local cortical gyrification. IEEE Trans. Med. Imag. 27 (2), 161-170.

Shaw, P., Greenstein, D., Lerch, J., Clasen, L., Lenroot, R., Gogtay, N., Evans, A., Rapoport, J., Giedd, J., 2006a. Intellectual ability and cortical development in children and adolescents. Nature 440 (7084), 676-679.

Shaw, P., Lerch, J., Greenstein, D., Sharp, W., Clasen, L., Evans, A., Giedd, J., Castellanos, F.X., Rapoport, J., 2006b. Longitudinal mapping of cortical thickness and clinical outcome in children and adolescents with attention-deficit/hyperactivity disorder. Arch. Gen. Psychiatry 63 (5), 540-549.

Shaw, P., Eckstrand, K., Sharp, W., Blumenthal, J., Lerch, J.P., Greenstein, D., Clasen, L., Evans, A., Giedd, J., Rapoport, J.L., 2007a. Attention-deficit/ hyperactivity disorder is characterized by a delay in cortical maturation. Proc. Natl. Acad. Sci. U. S. A. 104 (49), 19649-19654.

Shaw, P., Lerch, J.P., Pruessner, J.C., Taylor, K.N., Rose, A.B., Greenstein, D., Clasen, L. Evans, A., Rapoport, J.L., Giedd, J.N., 2007b. Cortical morphology in children and adolescents with different apolipoprotein e gene polymorphisms: an observational study. Lancet Neurol. 6 (6), 494-500.

Shaw, P., Kabani, N.J., Lerch, J.P., Eckstrand, K., Lenroot, R., Gogtay, N., Greenstein, D., Clasen, L., Evans, A., Rapoport, J.L., Giedd, J.N., Wise, S.P., 2008. Neurodevelopmental trajectories of the human cerebral cortex. J. Neurosci. 28 (14), 3586-3594.

Simon, T.J., Ding, L., Bish, J.P., McDonald-McGinn, D.M., Zackai, E.H., Gee, J., 2005. Volumetric, connective, and morphologic changes in the brains of children with chromosome 22q11.2 deletion syndrome: an integrative study. Neuroimage 25 (1), 169-180.

Sowell, E.R., Peterson, B.S., Thompson, P.M., Welcome, S.E., Henkenius, A.L., Toga, A.W., 2003. Mapping cortical change across the human life span. Nat. Neurosci. 6 (3), 309-315.

Thompson, P.M., Hayashi, K.M., Sowell, E.R., Gogtay, N., Giedd, J.N., Rapoport, J.L., de Zubicaray, G.I., Janke, A.L., Rose, S.E., Semple, J., Doddrell, D.M., Wang, Y., van Erp, T.G., Cannon, T.D., Toga, A.W., 2004. Mapping cortical change in alzheimer's disease, brain development, and schizophrenia. Neuroimage 23 (Suppl 1), 2-18.

van Amelsvoort, T., Daly, E., Robertson, D., Suckling, J., Ng, V., Critchley, H., Owen, M.J., Henry, J., Murphy, K.C., Murphy, D.G., 2001. Structural brain abnormalities associated with deletion at chromosome 22q11: quantitative neuroimaging study of adults with velo-cardio-facial syndrome. Br. J. Psychiatry 178, 412-419.

van Amelsvoort, T., Daly, E., Henry, J., Robertson, D., Ng, V., Owen, M., Murphy, K.C., Murphy, D.G., 2004. Brain anatomy in adults with velocardiofacial syndrome with and without schizophrenia: preliminary results of a structural magnetic resonance imaging study. Arch. Gen. Psychiatry 61 (11), 1085-1096.

van Amelsvoort, T., Zinkstok, J., Figee, M., Daly, E., Morris, R., Owen, M.J., Murphy, K.C., De Haan, L., Linszen, D.H., Glaser, B., Murphy, D.G., 2008. Effects of a functional comt polymorphism on brain anatomy and 
cognitive function in adults with velo-cardio-facial syndrome. Psychol. Med. 38 (1), 89-100.

Wilke, M., Sohn, J.H., Byars, A.W., Holland, S.K., 2003. Bright spots: correlations of gray matter volume with iq in a normal pediatric population. Neuroimage 20 (1), 202-215.
Zinkstok, J., Schmitz, N., van Amelsvoort, T., Moeton, M., Baas, F., Linszen, D., 2008. Genetic variation in comt and prodh is associated with brain anatomy in patients with schizophrenia. Genes Brain Behav. 7 (1), 61-69. 